\title{
A laparoscopic simulator - maybe it is worth making it yourself
}

\author{
Dominik A. Walczak,2, Piotr Piotrowski ${ }^{3}$, Adam Jędrzejczyk ${ }^{4}$, Dariusz Pawełczak², Zbigniew Pasieka² \\ ${ }^{1}$ Department of General Surgery, John Paul II Memorial Hospital, Belchatow, Poland \\ ${ }^{2}$ Department of Experimental Surgery, Medical University of Lodz, Lodz, Poland \\ ${ }^{3}$ Telecommunications and Teleinformatics Department, Technical University of Wroclaw, Wroclaw, Poland \\ ${ }^{4}$ Department of Urology, John Paul II Memorial Hospital, Belchatow, Poland
}

Videosurgery Miniinv 2014; 9 (3): 380-386

DOI: $10.5114 /$ wiitm.2014.44139

\begin{abstract}
Introduction: Laparoscopic trainers have gained recognition for improving laparoscopic surgery skills and preparing for operations on humans. Unfortunately, due to their high price, commercial simulators are hard to obtain, especially for young surgeons in small medical centers. The solution might be for them to construct a device by themselves.

Aim: To make a relatively cheap and easy to construct laparoscopic trainer for residents who wish to develop their skills at home.

Material and methods: Two laparoscopic simulators were designed and constructed: 1) a box model with an optical system based on two parallel mirrors, 2) a box model with an HD webcam, a light source consisting of LED diodes placed on a camera casing, and a modeling servo between the webcam and aluminum pipe to allow electronic adjustment of the optical axis.

Results: The two self-constructed simulators were found to be effective training devices, the total cost of parts for each not exceeding \$100. Advice is also given for future constructors.

Conclusions: Home made trainers are accessible to any personal budget and can be constructed with a minimum of practical skill. They allow more frequent practice at home, outside the venue and hours of surgical departments. What is more, home made trainers have been shown to be comparable to commercial trainers in facilitating the acquisition of basic laparoscopic skills.
\end{abstract}

Key words: laparoscopy, simulator, bench models, surgical training.

\section{Introduction}

Despite having a relatively short history, laparoscopy has become a commonly used and widespread surgical technique. Because of its many advantages, such as less postoperative pain, better cosmetic results, shorter hospital stay and earlier return to normal activities, it has become the gold standard in the treatment of various diseases [1].

However, compared with open surgical procedures, the laparoscopic approach is quite different and different skills are needed. The first is the ability to perform actions in three dimensions when only two can be observed on the monitor. The second is that the difference of depth perception, spatial relationship and long surgical instruments requires perfect eye-hand coordination. Moreover, the arms of the tools act as levers with a fulcrum at the site of the skin incision, and hence the real action with the tool handles is a mirror of the movement of the tool tips seen on the monitor [2].

For this reason, the training process is difficult and requires time and the opportunity to practice this kind of surgery. What is more, it demands the

\section{Address for correspondence}

Dominik A. Walczak MD, John Paul II Memorial Hospital, 123 Czalpiniecka St, 97-400 Belchatow, Poland, phone: +48 604436868 ,

e-mail: dr.dominikwalczak@gmail.com 
use of animal models or simulators. To date, most of these options are very expensive and not easily available for constant training.

\section{Aim}

Our goal was to build a relatively cheap and easyto-construct laparoscopic trainer for residents who want to develop their skills at home.

\section{Material and methods}

Two self-made laparoscopic simulators were designed and constructed:

\section{Mirror-based laparoscopic simulator}

The optical system of this simulator was designed using two parallel mirrors. The law of reflection states that the angle at which the beam is incident on the surface equals the angle at which it is reflected. Therefore, two mirrors were placed at a 45-degree angle (Photo $1 \mathrm{~B}$ ).

The housing for the laparoscopic trainer is a small plastic box with a fitted lid (Sortera, Ikea). The plastic wall is $2 \mathrm{~mm}$ thick and rigid, which is crucial for the stability of the construction. One of the side walls leans at a 45-degree angle, which helps to assemble the lower mirror $(30 \mathrm{~cm} \times 30 \mathrm{~cm})$.

Three holes were cut out from a lid. One $8 \mathrm{~cm}$ diameter hole was for the light source. Two $7 \mathrm{~cm}$ diameter holes were used for the trocars: these were fitted with $2 \mathrm{~mm}$ thick pieces of rubber sheet fixed with metal washers. This solution allows the movable ports to imitate the elasticity of the integument (Photo $1 \mathrm{C}$ ).

A $60 \mathrm{~cm}$ long oval furniture leg was fastened to the posterior part of the lid to form a slide for the upper mirror, which simulates a video monitor. The upper mirror $(20 \mathrm{~cm} \times 20 \mathrm{~cm})$ is fixed to the slide using two threaded rods, clamps and a 45-degree adjustable square. This system allows the upper mirror to move and adapt to the height of the user (Photo 1 A).

The base of the simulator is illuminated using a 48-diode LED bulb (GU-10, 230V, 2.6W) assembled with the light placed in the middle hole. The lighting system is covered from the outside by a small plastic box. All training pads may be inserted on the ramp (made from medium-density fiberboard - MDF) to provide a proper viewing angle (Photo $1 \mathrm{D}$ ).

\section{Webcam-based laparoscopic simulator}

This laparoscopic trainer was created using the same plastic box with a lid (Photo 2 A, B). As with the camera-less model, six holes were cut from the lid. In five holes, pieces of rubber sheet were placed in the same way as before (Photo 2 F). The middle, the largest aperture, was intended for camera placement, and was covered by a specially prepared, unzipped material sleeve. A Logitec Quickam pro 9000 webcam was used as the camera, with six LEDs placed on its casing as a light source (Photo $2 \mathrm{E}$ ). The webcam was connected through a servo to an aluminum pipe, allowing the optical axis to be changed electronically from direct to oblique. On the other end of the aluminum pipe, a control panel was placed to allow the optical axis and brightness to be controlled (Photo $2 \mathrm{G}$ ). During training, the camera may be placed in a stand, which was previously used as a lamp base (Photo $2 \mathrm{C}$ ).

\section{Results}

The costs of parts for both laparoscopic simulators are presented in Table I. The prices are given in Polish currency. The average rate of exchange at the time of construction was PLN 3.27 for \$1 and PLN 4.17 for $€ 1$. The total cost of the camera-less laparoscopic simulator was $\$ 51 / € 40$, while the webcam-based laparoscopic simulator was \$99/€78.

Since some parts such as screws, nuts, timber slats, plastic box or material sleeve are commonly available in every household or can be made easily, their prices were not taken into consideration in evaluating the cost.

\section{Discussion}

Laparoscopic trainers have gained recognition for improving the skills of laparoscopic surgery and acting as preparation for operations on humans [3-5]. Unfortunately, due to their high price, commercial simulators are hard to afford, especially for young surgeons in small medical centers. The solution might be for them to construct a device by themselves.

The design of the first trainer is based on mirrors. It is undoubtedly a cheaper model which doesn't need a computer and provides adequate training conditions. A study by Bruynzeel et al. demonstrated that the mirror trainer and the camera trainer are equally effective in teaching laparoscopic suturing 

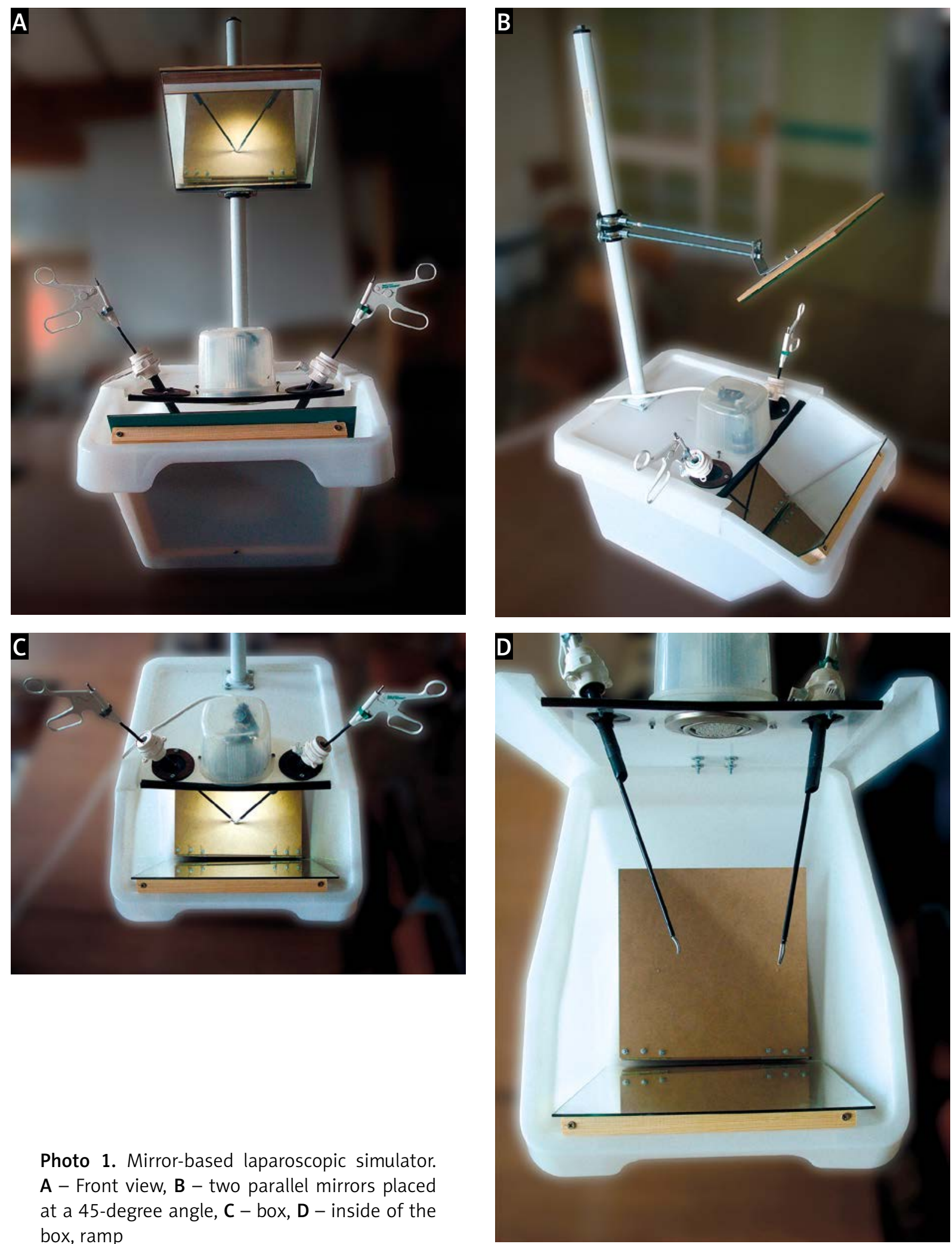

Photo 1. Mirror-based laparoscopic simulator. A - Front view, B - two parallel mirrors placed at a 45-degree angle, $\mathbf{C}$ - box, $\mathbf{D}$ - inside of the box, ramp 

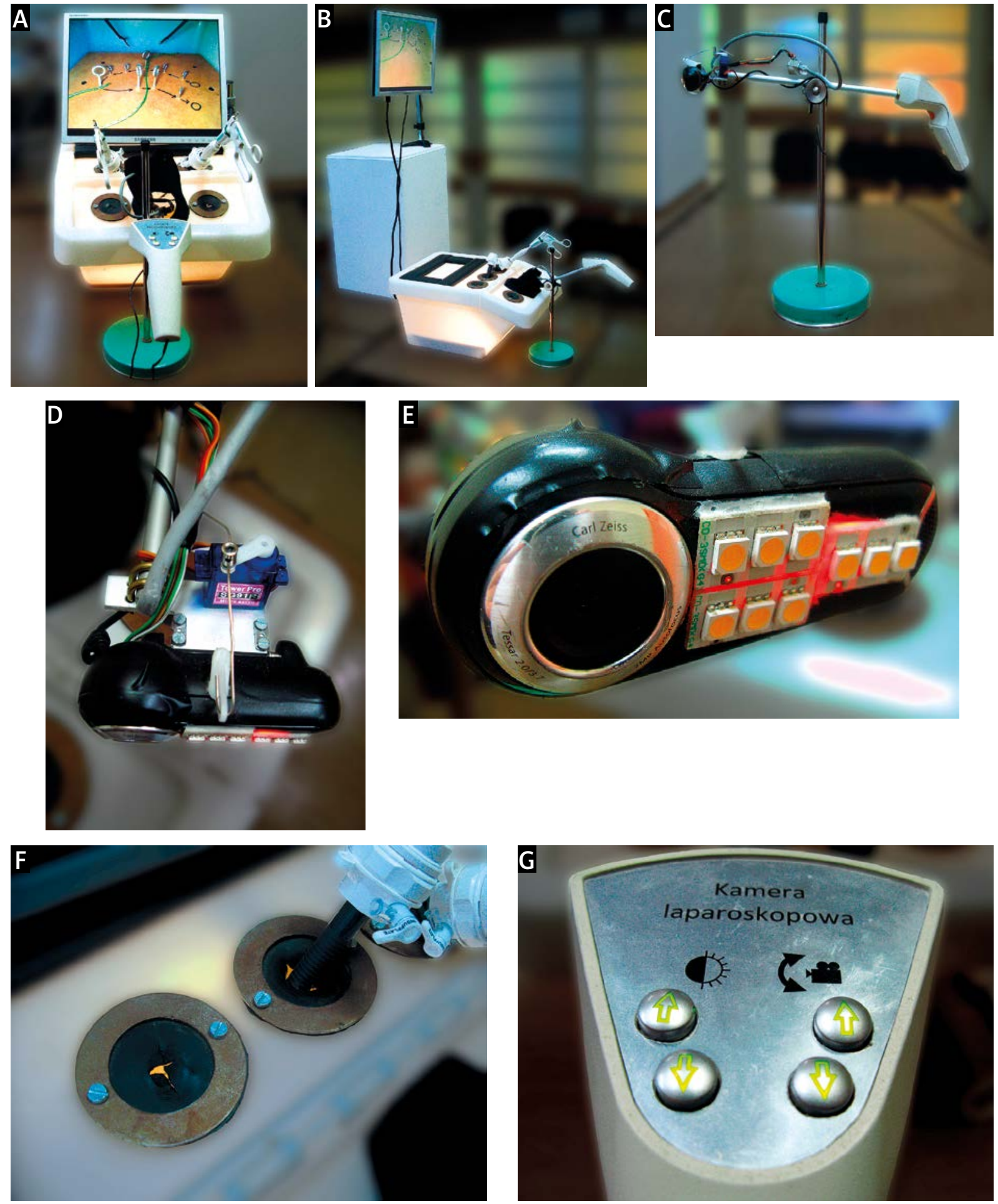

Photo 2. Webcam-based laparoscopic simulator. A, B - Simulator, C - camera placed in a stand, D - connection between an aluminum pipe and the webcam through servo, $\mathbf{E}$ - webcam with LEDs placed on its casing, $\mathbf{F}$ - holes for the trocars, $\mathbf{G}$ - the control panel 
Table I. Cost of parts for both laparoscopic simulators

\begin{tabular}{|c|c|c|c|}
\hline Camera-less laparoscopic simulator & PLN & Webcam-based laparoscopic simulator & PLN \\
\hline Box with a lid & 29.99 & Box with a lid & 29.99 \\
\hline Mirror $20 \times 20$ & 8 & Washer (6 pcs) & 18 \\
\hline Mirror $30 \times 30$ & 12 & Rubber sheet $(1 \times 1 \mathrm{~m})$ & 32.75 \\
\hline Washer (2 pcs) & 6 & Aluminum pipe (1 m) & 3 \\
\hline Clamp (2 pcs) & 8.60 & Webcam & 208 \\
\hline Threaded rod (1 meter) & 2.40 & LED diodes (6 pcs) & 4.8 \\
\hline 45-degree adjustable square & 1.55 & Power supply adaptor & 30 \\
\hline LED bulb & 20 & Optional & \\
\hline Light fitting & 25 & & \\
\hline Medium-density fiberboard (2 pcs $25 \times 25 \mathrm{~cm})$ & 2.37 & Button (4 pcs) & 24 \\
\hline Furniture leg & 13.50 & Enclosure & 79 \\
\hline Rubber sheet $(1 \times 1 \mathrm{~m})$ & 32.75 & Servo & 17 \\
\hline Electric wire (2 m) & 5.70 & & \\
\hline Screws, nuts, small plastic box, timber slat & - & Screws, nuts, unzipped material sleeve & - \\
\hline Total cost & 167.86 & $\begin{array}{c}\text { Total cost } \\
\text { With optional equipment }\end{array}$ & $\begin{array}{l}326.54 \\
446.54\end{array}$ \\
\hline
\end{tabular}

skills [6]. It is noteworthy that mirror simulators available on the market are incomparably more expensive and they cost from $\$ 300$ to as much as $\$ 2000$ (Simulab Corporation) [7]. Only one other self-made, mirror-based laparoscopic simulators can be found in medical databases (Medline). This construction, designed by Robinson and Kusher, also uses two mirrors which reflect the image from the box; however, the placement of the mirrors differs from our model [8].

The second trainer, based on a camera, replicates real laparoscopy far more than a mirror-based trainer. So far, several similar models have been designed and created by other authors. The easiest solution was introduced by Pawar, who used a digital camera connected with a television set [9]. In one report, a spy camera was mounted on a movable arm [10]. The webcam is the most popular solution, as it is more practical than a spy camera or video camera, which needs a television monitor: our webcam-based trainer box can easily be connected to a laptop or desktop computer, which is more likely to be available for individual use. Moreover, webcams are generally easily affordable compared with camcorders and spy cameras. However, the techni- cal specifications of the webcam are important: HD webcams are highly recommended because they provide a clear picture without blurring the moving instruments [11].

The are different ways of mounting a webcam inside the trainer. They could be fixed to the internal part of a lid [11, 12] or placed on the inner elevation as suggested by Beatty [13]. In our opinion, the best solution is to mount a webcam to a pipe, as this not only allows the camera to move in all planes, but also imitates the operation of a real laparoscope and accustoms the user to conditions during an operation, which might be helpful for inexperienced surgeons. Moreover, the moveable arm could be placed in a stand during practice, negating the need for an assistant to hold the camera.

The material used to construct the simulator is not insignificant. A paper box [14], timber case [15], metal basket [12] and plastic container [10] were previously described as the housing for the trainer. The fact that the box is made of durable plastic means that it is both appropriate for holding organic tissues and easy to clean.

Future constructors of a similarly designed laparoscopic simulator may find the following sugges- 
tions helpful. The first is to place LED diodes on the webcam casing: by doing this, the axis of view of the camera rarely matches the illumination axis and so there are no shadows. The presence of the lamp as a light source, or a box transparent to light, allows the trainee to use shadow as additional spatial information. While it undoubtedly simplifies the performance of tasks, it differs from classical laparoscopy $[2,16]$.

The second innovation is the incorporation of a movable connection between the webcam and the aluminum pipe. In our trainer, it is created through a modeling servo. The whole mechanism is controlled electronically. This solution allows the optical axis to be changed easily from direct to oblique. Researchers from Dundee University directed by Alfred Cuschieri revealed that the angle created between the axis of view and the axis of tool insertion has a significant influence on the accuracy and velocity of tool movements in the two-dimensional laparoscopic field [17]. This feature better accustoms the user to some of the handicaps and inconveniences of an oblique view. We realize that the introduction of this modification requires basic electronic knowledge; however, it should be considered because it improves exercise realism.

The next noteworthy solution is the way that the tool is inserted inside the simulator. In trainers designed by other authors, laparoscopic tools are usually placed in small holes drilled in the lid $[9,10,13]$. For the simulator presented in this study, $2 \mathrm{~mm}$ thick pieces of rubber sheet were placed in $7 \mathrm{~cm}$ apertures to mirror the elasticity of the abdominal wall. These rubber discs had cross incisions in the middle to allow trocars to be inserted through them.

Most laparoscopic instruments necessary for training are disposable and, after being properly sterilized, are suitable for training. Similarly, expired instruments are useful for this purpose. The only nondisposable instrument that is still necessary is the needle holder. Unfortunately, graspers are poor substitutes for this device because they do not maintain the grip on needles sufficiently to drive it into the "tissues" and tie the knot [18]. The cheapest brand new needle holders can be purchased from online auctions such as eBay for approximately $\$ 100$.

Self-made simulators allow basic laparoscopic skills to be practiced. The items used to create the simulated task are generally cheap and can easily be found in hypermarkets [18]. The apparatus allows tasks to be performed which develop common skills such as instrument navigation, coordination, grasping, lifting, cutting, clip application or intracorporeal knot suturing [19]. For example, handling skills can be developed by transferring matches from one plate to another, while depth perception and handeye coordination can be improved by running thread through holes of different size, placed at different levels and angles, or by cutting out a shape along a drawn line from a rubber glove. Colored rubber tubes can simulate vessels, while a water-filled glove can replicate a gall bladder or ovarian cyst $[18,20$, 21]. The range of exercises is limited only by the imagination.

Moreover, low-cost box trainers allow the key part of some surgical or urological operations to be performed. Self-made models simulating laparoscopic choledochotomy, division and ligation of the appendicular artery and appendix, mesh placement during hernia repair, splenectomy, and the closure of enterotomy or urethrovesical anastomoses have been described. Simulations might be carried out with high-resolution photographs of the anatomical region taken from real laparoscopic procedures, and photographs could be placed in the background to provide a clinical and anatomical context [22, 23].

\section{Conclusions}

Several studies have compared commercial laparoscopic trainers and less expensive home-made laparoscopic trainers. A meta-analysis performed by Nguyen et al. showed that they are equally proficient in facilitating the acquisition of basic laparoscopic skills [24].

\section{References}

1. Bittner R. Laparoscopic surgery - 15 years after clinical introduction. World J Surg 2006; 30: 1190-203.

2. Grobelski B, Walczak DA, Pasieka Z. New ways of visualization in laparoscopic surgery. Videosurgery Miniinv 2010; 5: 120-8.

3. Łaski D, Stefaniak TJ, Makarewicz Z, et al. New comprehensive surgical curriculum of pre-graduate surgical education. Videosurgery Miniinv 2013; 8: 200-10.

4. Willaert W, Van De Putte D, Van Renterghem K, et al. Training models in laparoscopy: a systematic review comparing the effectiveness in learning surgical skills. Acta Chir Belg 2013; 113: 77-95.

5. Łaski D, Stefaniak TJ, Makarewicz Z, et al. Structuralized boxtrainer laparoscopic training significantly improves performance in complex virtual reality laparoscopic tasks. Videosurgery Miniinv 2012; 7: 27-32. 
6. Bruynzeel H, de Bruin AF, Bonjer HJ, et al. Desktop simulator: key to universal training? Surg Endosc 2007; 21: 1637-40.

7. Available at: http://www.simulab.com/products/surgery/laparoscopic

8. Robinson JK, Kushner DM. Development and validation of a home-based, mirrored, gynecologic laparoscopy trainer. J Minim Invasive Gynecol 2006; 13: 102-7.

9. Pawar DS, Singh SK, Benjwal S, Kumari I. A novel idea of using digital camera for laparoscopy training in urology. Urol J 2010; 7: 56-8.

10. Pokorny MR, McLaren SL. Inexpensive home-made laparoscopic trainer and camera. ANZ J Surg 2004; 74: 691-3.

11. Khine M, Leung E, Morran C, Muthukumarasamy G. Homemade laparoscopic simulators for surgical trainees. Clin Teach 2011; 8: 118-21.

12. Jaber $\mathrm{N}$. The basket trainer: a homemade laparoscopic trainer attainable to every resident. J Minim Access Surg 2010; 6: 3-5.

13. Beatty JD. How to build an inexpensive laparoscopic webcam-based trainer. BJU Int 2005; 96: 679-82.

14. Chung SY, Landsittel D, Chon CH, et al. Laparoscopic skills training using a webcam trainer. J Urol 2005; 173: 180-3.

15. Sparks DA, Chase DM, Lee WS. An inexpensive solution for laparoscopic simulation OPUS 12 Scientist 2008; 2: 1-3.

16. Breedveld P, Stassen HG, Meijer DW, Jakimowicz JJ. Observation in laparoscopic surgery: overview of impeding effects and supporting aids. J Laparoendosc Adv Surg Tech A 2000; 10: 231-41.

17. Patil PV, Hanna GB, Cuschieri A. Effect of the angle between the optical axis of the endoscope and the instruments' plane on monitor image and surgical performance. Surg Endosc 2004; 18: 111-4.

18. Gravante G, Venditti D. A systematic review on low-cost box models to achieve basic and advanced laparoscopic skills during modern surgical training. Surg Laparosc Endosc Percutan Tech 2013; 23: 109-20.

19. Clevin L, Grantcharov TP. Does box model training improve surgical dexterity and economy of movement during virtual reality laparoscopy? A randomised trial. Acta Obstet Gynecol Scand 2008; 87: 99-103.

20. Chandrasekera SK, Donohue JF, Orley D, et al. Basic laparoscopic surgical training: examination of a low-cost alternative. Eur Urol 2006; 50: 1285-90, 1290-1.

21. Hasson HM, Kumari NV, Eekhout J. Training simulator for developing laparoscopic skills. JSLS 2001; 5: 255-65.

22. Hwang J, Telem DA, Nguyen SQ, et al. A novel laparoscopic ventral herniorrhaphy training system. Surg Laparosc Endosc Percutan Tech 2010; 20: e16-8.

23. Jiang C, Lin T, Zhang C, et al. A training model for laparoscopic urethrovesical anastomosis. J Endourol 2008; 22: 1541-5.

24. Nguyen T, Braga LH, Hoogenes J, Matsumoto ED. Commercial video laparoscopic trainers versus less expensive, simple laparoscopic trainers: a systematic review and meta-analysis. J Urol 2013; 190: 894-9.

Received: 26.10.2013, accepted: 17.02.2014. 